\title{
GEOLOGÍA Y MINERALOGÍA DE YACIMIENTOS DE CAOLÍN EN EL DEPARTAMENTO DE BOYACÁ
}

(Kaolin's Characterization and Potential in the Boyacá Department)

Sandra Rocío Manosalva Sánchez*, Wilson Enario Naranjo Merchán**

*Docente Escuela de Ingeniería Geológica, UPTC-Sogamos, Grupo de Investigación Ingeniería Geológica, smanosalva@sogamoso.uptc.edu.co - **Consultor, wilsonnaranjo@hotmail.com

(Recibido Junio 19 de 2006 y aceptado Marzo 22 de 2007)

\begin{abstract}
Resumen:
En la cordillera Oriental de Colombia, zona centro, se presenta un potencial minero para la extracción de arcillas blancas, en su mayoría depósitos de origen sedimentario, de tipo lacustre y coluvial. Se conocen yacimientos de caolín en Tunja, Siachoque, Tutasá, Gachantivá y Arcabuco; en estos dos últimos municipios están los yacimientos más importantes por su extensión y por la minería que allí se desarrolla hace más de 50 años para la fabricación de cerámica. En este artículo se plantea una metodología para el estudio de las arcillas en todas las fases del desarrollo de un proyecto minero, comenzando por la exploración y terminando por la obtención de un mineral de alta pureza.
\end{abstract}

Palabras clave: Arcillas, Boyacá, Mineralogía, Caolín, Difracción.

\section{Abstract:}

In the Colombian Oriental Mountain range, central region, there is a mining potential for the extraction of white clays, its majority of sedimentary origin, and the deposits are of lacustrine and co-alluvial type. The kaolin locations are known in: Tunja, Siachoque, Tutasá, Gachantivá and Arcabuco municipalities. In the last two there are the most important locations by their extension and for being the mining sites that have been developed during more than for 50 years into the ceramic production. It is stated a methodology to study the clays in all the development phases of a mining project, beginning with the exploration and accomplishing a high purity mineral.

Key Words: Clays, Boyacá, Mineralogy, Kaolin, Diffraction.

\section{INTRODUCCIÓN}

$\mathbf{L}$ a investigación surgió de la necesidad de los sectores gubernamental, industrial y académico de conocer el potencial minero de las arcillas existentes en el departamento de Boyacá, solo identificable a través de su composición química y mineralógica.

La mineralogía de arcillas es una ciencia relativamente joven; el concepto de mineral arcilloso se desarrolló desde 1930 (Grim, 1962). En Colombia el estudio detallado, sistemático y continuo de las arcillas hasta ahora comienza. Existen algunos estudios sobre el origen de los minerales arcillosos (Rodríguez, 1998), por lo que este proyecto sirve como marco de referencia.

En la Cordillera Oriental, zona centro, se presenta un potencial minero para la extracción de arcillas blancas, en su mayoría depósitos de origen sedimentario, de tipo lacustre y coluvial. Son conocidos los yacimientos de caolín en Tunja, Siachoque, Tutasá, Gachantivá y Arcabuco; en estos dos últimos municipios están los yacimientos más importantes por su extensión y por la minería que allí se desarrolla desde hace más de 50 años para la fabricación de cerámica.

La caracterización química y mineralógica de un yacimiento parte del conocimiento geológico del depósito, ya que es a través de este como se escoge el tipo de muestreo por realizar y se determinan las mejores alternativas de explotación. Es así como en las zonas de estudio, las arcillas blancas de origen sedimentario fueron caracterizadas a partir de muestras representativas de cada uno de los horizontes de alteración presentes en los frentes de explotación, distinguibles por sus coloraciones, reflejo de la presencia de materia orgánica y de minerales accesorios como limonita, lepidocrocita y leucoxeno; horizontes que hacen recomendable una extracción selectiva en el yacimiento.

La caracterización mineralógica de arcillas ha de hacerse siempre utilizando técnicas analíticas combinadas, ya que no existe un solo método que suministre toda la información de composición y textura en el depósito; a nivel mundial, la difractometría de rayos X (Thorez, 2003) es el análisis más utilizado y completo en la identificación de minerales arcillosos, sin embargo, para este estudio fue necesario conocer la relación con los otros minerales presentes en el depósito, a través de análisis óptico; en consecuencia, la difractometría de rayos X y el análisis óptico, en conjunto, permitieron identificar tipo, forma y distribución de los minerales arcillosos y de la arcilla.

Los minerales arcillosos más abundantes en estos yacimientos son illita y caolinita; esta última siempre en cantidades superiores al $20 \%$, susceptible de beneficiarse y de ser concentrada, 
por lo que industrialmente es llamada caolín. Todas las muestras estudiadas presentan patrones muy similares en los diferentes análisis de laboratorio, indicadores de los mismos minerales dominantes: illita, caolinita y cuarzo, minerales arci1losos mal cristalizados, producto de la acidez del medio que provoca su degradación. Los minerales accesorios (menores del 5\%) que se presentan son: sericita, rutilo, anatasa, leucoxeno, lepidocrocita, goethita, limonita, circón y turmalina.

\section{MATERIALES Y MÉTODOS}

Los yacimientos estudiados se encuentran ubicados en los municipios de Tunja, Siachoque, Tutasá, Gachantivá y Arcabuco, departamento de Boyacá (ver figura 1); en el municipio de Arcabuco se escogió como área piloto para la aplicación de toda la metodología, en el estudio de minerales arcillosos, la mina La Esperanza.

Para el desarrollo metodológico se tomó como base el proceso para el establecimiento de minerales industriales (Wilson, 1994), ajustándolo a los objetivos del proyecto de investigación (ver figura 2).

La primera etapa de reconocimiento geológico se realizó mediante revisión fotogeológica y observación en campo del área de estudio; se identificaron las unidades geológicas presentes y se realizó la cartografía geológica a escala 1:10.000, delimitando los depósitos de arcillas y realizando el levantamiento estratigráfico a detalle en los frentes de explotación, a través del cual se determinaron espesores, intercalaciones, continuidad lateral y se seleccionaron los puntos para muestreo. El plano geológico de referencia fue la plancha J-12 Tunja (Renzoni 1976), a escala 1:100.000.
En la segunda etapa se analizaron química y mineralógicamente las arcillas, a partir de muestras colectadas en los frentes de explotación y zonas de acopio; en los frentes de explotación se hicieron canales, en donde se tomaron muestras representativas para cada uno de los niveles, diferenciables en campo por su coloración (ver figura 3). Se realizaron análisis por petrografía, difracción de rayos $\mathrm{X}$ en muestras orientadas y en polvo, microscopía electrónica y químico por espectrometría de fluorescencia de rayos $\mathrm{X}$; partiendo de estos análisis se identificaron los minerales presentes, su proporción relativa, así como su forma, tamaño y grado de cristalinidad; estableciendo relaciones entre los minerales y sus elementos químicos marcadores, obteniendo tendencias de incremento, disminución o estabilidad en los contenidos de caolinita en los frentes de explotación.

En una tercera etapa se realizó el beneficio en el laboratorio, mediante procesos de dispersión, tamizado, sedimentación y flotación; se analizaron los productos del tamizado y sedimentación por petrografía y de la flotación por petrografía, difracción de rayos $\mathrm{X}$ y análisis químico por espectrometría de fluorescencia de rayos $X$. La caracterización química $y$ mineralógica de los productos del beneficio, especialmente de las espumas, producto de flotación, permitió evaluar la calidad del proceso, mediante comparaciones de estos análisis con los ya realizados en las muestras naturales.

Por último, en la cuarta etapa se realizó una evaluación de las propiedades físicas, para posibles usos industriales, de acuerdo con las especificaciones técnicas de clientes potenciales como la industria cerámica, del papel y la pintura, entre otras, en los cuales la arcilla, en especial el caolín, es materia prima.
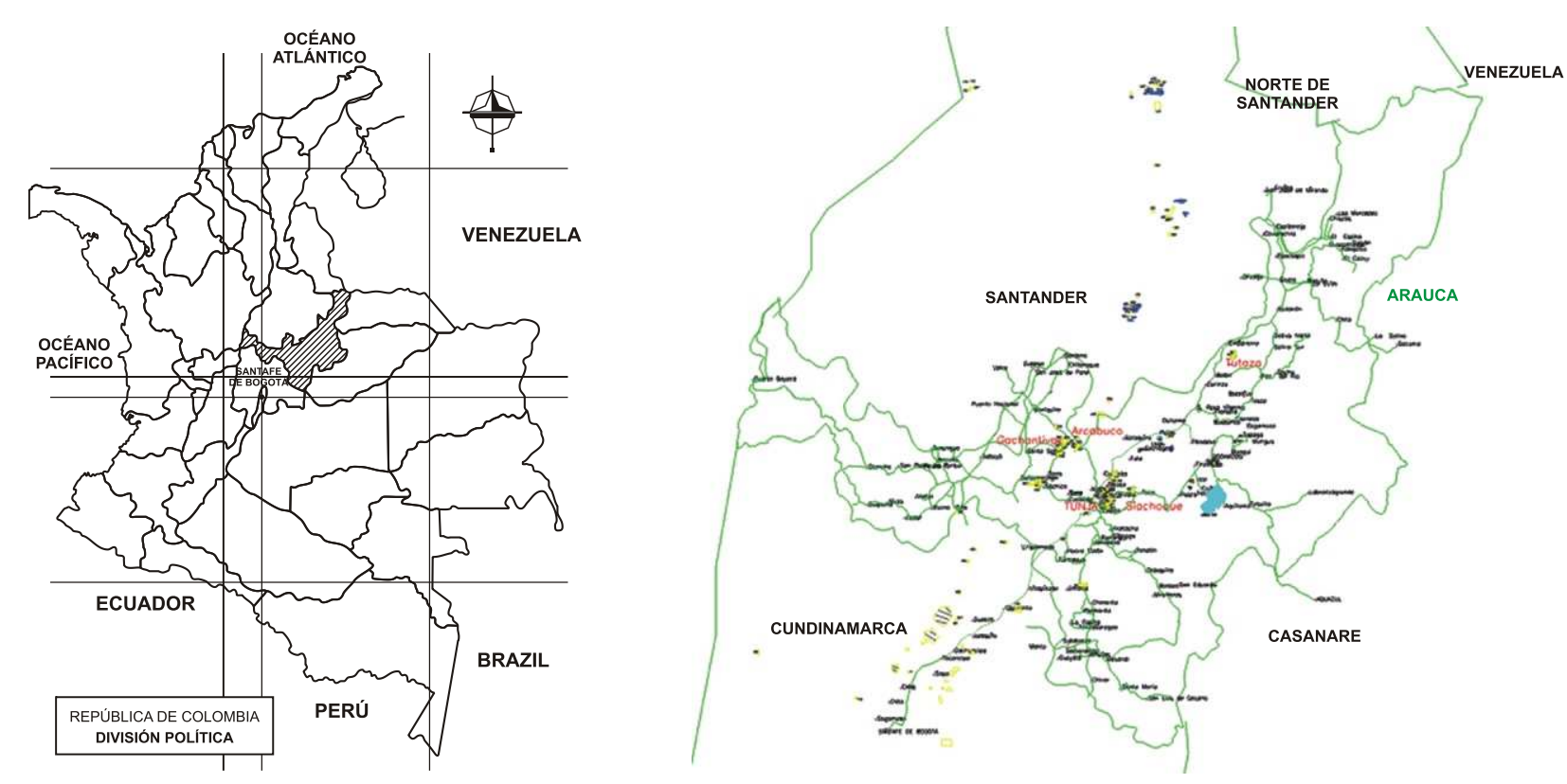

Figura 1. Localización geográfica. Fuente: IGAC, 1984 


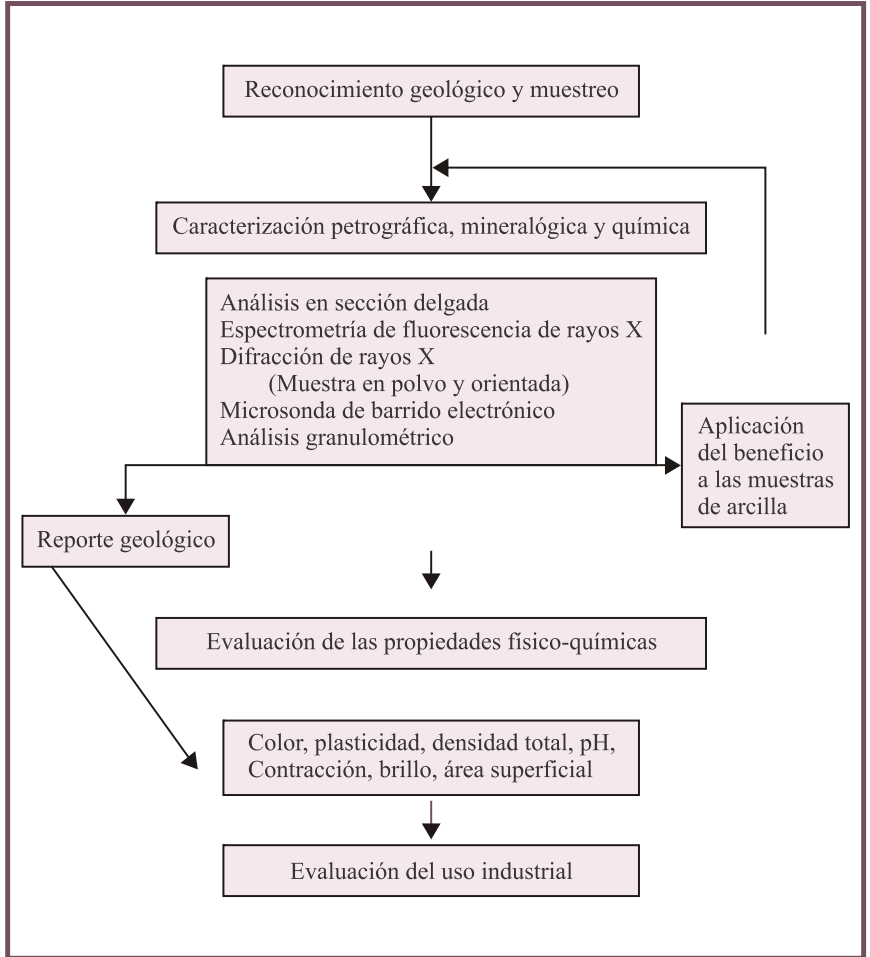

Figura 2. Diagrama de flujo para la caracterización mineralógica de las arcillas. Fuente: Manosalva (2005).

\section{DISCUSIÓN}

El yacimiento ubicado en el municipio de Tutasá (Boyacá), entre los kilómetros 6 y 7 en el carreteable que de Belén conduce al municipio de Soatá, tiene una extensión de 474 ha. Este depósito está sobre rocas de la Formación Cuarzomonzonita de Santa Rosita (Pdcs), rocas que datan del Silúrico. Los minerales arcillosos identificados fueron caolinita e illita, y de la arcilla el cuarzo. En el depósito se llevan a cabo labores de explotación características de pequeña y rudimentaria minería.

El yacimiento de Chivatá se ubica en la vereda Manarrica; entre los kilómetros 5 y 6 en el carreteable que de Chivatá conduce al municipio de Toca. El depósito de caolín yace sobre rocas que datan del Cretácico, específicamente sobre la Formación Plaeners (kg2). Mineralogicamente se presentan caolinitas mal critalizada; una mezcla principalmente de caolinita, illita y óxidos de hierro.

Los yacimientos de Arcabuco y Gachantivá son de origen sedimentario, de tipo coluvial (Qcr), contienen materiales deslizados de la Formación Ritoque, a la cual suprayace, constituida por arcillolitas y lodolitas de color crema a pardo oscuro con presencia de materia orgánica (raíces y troncos bien preservados), abundantes hacia la base (ver figura 4).

La arcilla fue clasificada composicionalmente como: Arcilla illítico-caolinítica, con abundante cuarzo. El yacimiento de Siachoque es el de mayor concentración de caolinita.

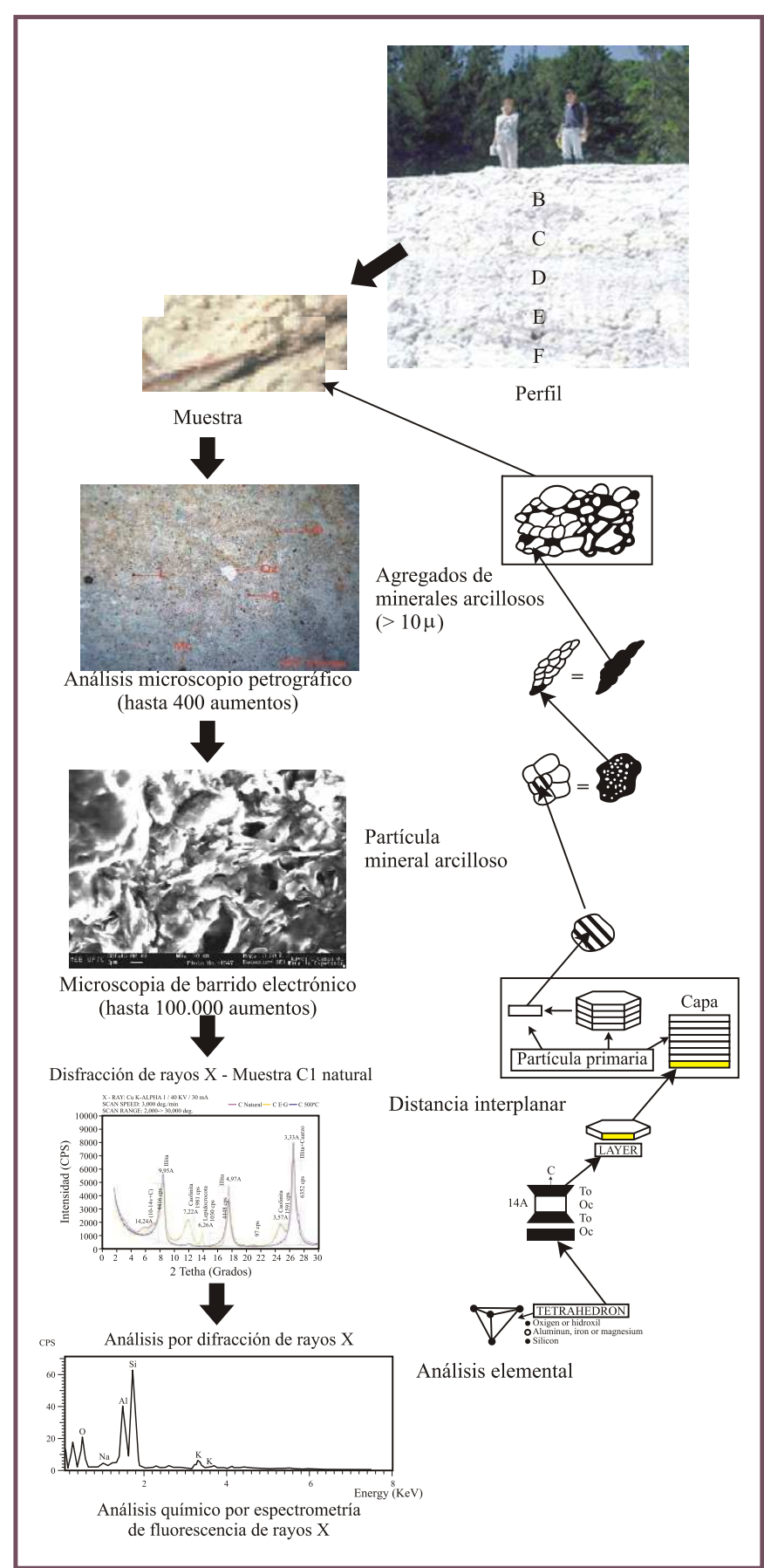

Figura 3. Proceso metodológico en la caracterización mineralógica y química de la arcilla para cada una de las muestras tomadas en los niveles de los frentes de explotación.

De la caracterización en todos los yacimientos se pudo establecer que las arcillas presentan patrones muy similares en los diferentes análisis de laboratorio, indicadores de los mismos minerales dominantes: illita, caolinita y cuarzo, identificados a través de la petrografía, análisis químico y de difracción de rayos $\mathrm{X}$, siendo importante destacar la mala cristalización de los minerales arcillosos y comportamientos vermiculítico- 


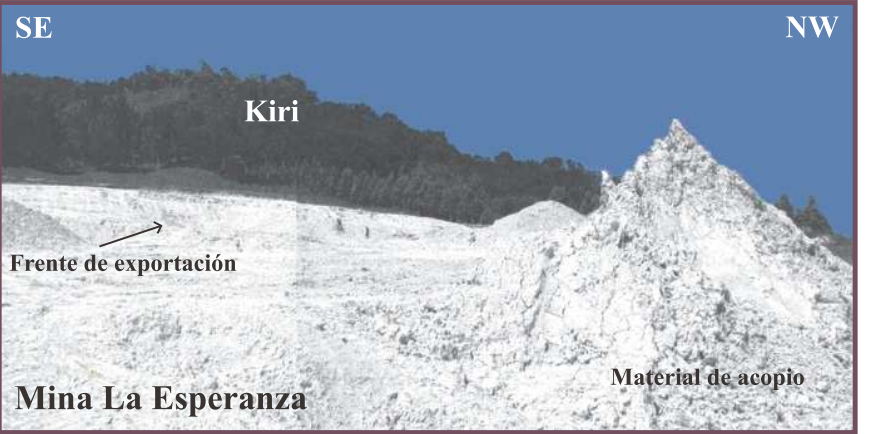

Figura 4. Frente de explotación de la mina

"La Esperanza", Arcabuco, Boyacá.

clorítico de la illita. La caolinita está entre un 20 y un 50\% (ver figura 4).

La caolinita, mineral de interés comercial no está bien cristalizada (desordenada); por su origen sedimentario, esta mala cristalinidad, que se manifiesta en la difracción de rayos $\mathrm{X}$ de muestras orientadas, mediante la baja simetría que presenta la reflexión de $7 \AA ̊$ hacia los ángulos bajos, y la de $3,5 \AA$ hacia los ángulos altos, y en la mayoría de las muestras analizadas en el microscopio electrónico, se presentó de manera muy difusa, casi amorfa.

Los minerales accesorios (menores del 5\%), identificados a través de la petrografía son: sericita, rutilo, anatasa, leucoxeno, goethita, limonita, circón y turmalina; la mayoría de estos minerales se presentan en la fracción de limo fino entre 9 y 30 micras, a excepción del rutilo, que está ampliamente distribuido en una fracción fina menor a 2 micras, y de la lepidocrocita y limonita, que está impregnando los minerales arcillosos. Estos minerales accesorios marcan la diferencia de un depósito a otro, especialmente la limonita y goethita, que es más abundante en los yacimientos de Tunja, y, en general, en todos los depósitos se acumulan los óxidos de hierro, en los niveles inferiores junto con el material orgánico.

A partir de la caracterización química y mineralógica se estableció que para elevar el tenor de caolinita se debía disminuir el contenido de illita y cuarzo, así como de rutilo, leucoxeno, anatasa, lepidocrocita y limonita, minerales estos denominados como impurezas y responsables de las coloraciones en la arcilla. Por tanto, con el fin de concentrar la mayor cantidad de caolinita, el procesamiento en húmedo fue el escogido para tratar las arcillas de la mina La Esperanza, en donde se siguieron las siguientes etapas: dispersión, tamizado, sedimentación y flotación. Se determinó que el proceso de beneficio, especialmente la flotación, disminuye el contenido de titanio, representado por leucoxeno y rutilo.

Con la información sobre las propiedades físicas y la caracterización mineralógica y química del material arcilloso, se procedió a evaluar su(s) uso(s) industrial(es), de conformidad con las especificaciones establecidas en la norma ICONTEC 2428 para el mercado de las arcillas blancas a nivel nacional. Se estableció que las arcillas presentan características que se ajustan a los requerimientos en las industrias del caucho y la cerámica, pero para otras aplicaciones en las que la arcilla tiene un mayor valor agregado, como recubrimiento para el papel, cemento blanco y pinturas, no sirve, ni en bruto, ni con el proceso de beneficio que se realizó. En el caso de la cerámica, esta arcilla presenta índices de plasticidad bajos; sin embargo, con el mejoramiento del proceso de beneficio y consecuente eliminación de cuarzo, illita y óxidos de hierro y titanio, esta tiende a mejorar y cumplir con las especificaciones. Existen algunos horizontes, especialmente los superiores, que por su coloración blanca, tanto al natural como calentada, se pueden utilizar en la fabricación de azulejos (revestimiento poroso), sanitarios, gres cerámico y vajilla. La presencia de cuarzo de forma abundante en la arcilla no genera un inconveniente en la utilización para la cerámica, ya que hace parte de la materia prima, utilizándose como desengrasante, haciendo parte del vidriado, elevando la porosidad de los productos, facilitando el secado y desgasificación de las piezas cocidas, además de 4. CONCLUSIONES
Las arcillas explotadas son de origen sedimentario, de tipo
coluvial y lacustre; contienen materiales deslizados de las
Formaciones circundantes, y suprayacen a estas. Se clasifi-
caron las arcillas como illítico-caoliníticas, donde minerales
como illita, caolinita y cuarzo son los más abundantes,
presentando una mala cristalización.
La caracterización química y mineralógica permitió establecer
el tipo de proceso de beneficio, frente a problemas específicos
como, por ejemplo, la presencia de óxidos de hierro que
impregnan y colorean las arcillas.
La caracterización mineralógica de los productos del beneficio
permitió concluir que estos procesos pueden afectar la
cristalinidad de los minerales. En el caso específico de la mina 4. CONCLUSIONES
Las arcillas explotadas son de origen sedimentario, de tipo
coluvial y lacustre; contienen materiales deslizados de las
Formaciones circundantes, y suprayacen a estas. Se clasifi-
caron las arcillas como illítico-caoliníticas, donde minerales
como illita, caolinita y cuarzo son los más abundantes,
presentando una mala cristalización.
La caracterización química y mineralógica permitió establecer
el tipo de proceso de beneficio, frente a problemas específicos
como, por ejemplo, la presencia de oxidos de hierro que
impregnan y colorean las arcillas.
La caracterización mineralógica de los productos del beneficio
permitió concluir que estos procesos pueden afectar la
cristalinidad de los minerales. En el caso específico de la mina 4. CONCLUSIONES
Las arcillas explotadas son de origen sedimentario, de tipo
coluvial y lacustre; contienen materiales deslizados de las
Formaciones circundantes, y suprayacen a estas. Se clasifi-
caron las arcillas como illítico-caoliníticas, donde minerales
como illita, caolinita y cuarzo son los más abundantes,
presentando una mala cristalización.
La caracterización química y mineralógica permitió establecer
el tipo de proceso de beneficio, frente a problemas específicos
como, por ejemplo, la presencia de óxidos de hierro que
impregnan y colorean las arcillas.
La caracterización mineralógica de los productos del beneficio
permitió concluir que estos procesos pueden afectar la
cristalinidad de los minerales. En el caso específico de la mina 4. CONCLUSIONES
Las arcillas explotadas son de origen sedimentario, de tipo
coluvial y lacustre; contienen materiales deslizados de las
Formaciones circundantes, y suprayacen a estas. Se clasifi-
caron las arcillas como illítico-caoliníticas, donde minerales
como illita, caolinita y cuarzo son los más abundantes,
presentando una mala cristalización.
La caracterización química y mineralógica permitió establecer
el tipo de proceso de beneficio, frente a problemas específicos
como, por ejemplo, la presencia de óxidos de hierro que
impregnan y colorean las arcillas.
La caracterización mineralógica de los productos del beneficio
permitió concluir que estos procesos pueden afectar la
cristalinidad de los minerales. En el caso específico de la mina 4. CONCLUSIONES
Las arcillas explotadas son de origen sedimentario, de tipo
coluvial y lacustre; contienen materiales deslizados de las
Formaciones circundantes, y suprayacen a estas. Se clasifi-
caron las arcillas como illítico-caoliníticas, donde minerales
como illita, caolinita y cuarzo son los más abundantes,
presentando una mala cristalización.
La caracterización química y mineralógica permitió establecer
el tipo de proceso de beneficio, frente a problemas específicos
como, por ejemplo, la presencia de óxidos de hierro que
impregnan y colorean las arcillas.
La caracterización mineralógica de los productos del beneficio
permitió concluir que estos procesos pueden afectar la
cristalinidad de los minerales. En el caso específico de la mina 4. CONCLUSIONES
Las arcillas explotadas son de origen sedimentario, de tipo
coluvial y lacustre; contienen materiales deslizados de las
Formaciones circundantes, y suprayacen a estas. Se clasifi-
caron las arcillas como illítico-caoliníticas, donde minerales
como illita, caolinita y cuarzo son los más abundantes,
presentando una mala cristalización.
La caracterización química y mineralógica permitió establecer
el tipo de proceso de beneficio, frente a problemas específicos
como, por ejemplo, la presencia de óxidos de hierro que
impregnan y colorean las arcillas.
La caracterización mineralógica de los productos del beneficio
permitió concluir que estos procesos pueden afectar la
cristalinidad de los minerales. En el caso específico de la mina 4. CONCLUSIONES
Las arcillas explotadas son de origen sedimentario, de tipo
coluvial y lacustre; contienen materiales deslizados de las
Formaciones circundantes, y suprayacen a estas. Se clasifi-
caron las arcillas como illítico-caoliníticas, donde minerales
como illita, caolinita y cuarzo son los más abundantes,
presentando una mala cristalización.
La caracterización química y mineralógica permitió establecer
el tipo de proceso de beneficio, frente a problemas específicos
como, por ejemplo, la presencia de óxidos de hierro que
impregnan y colorean las arcillas.
La caracterización mineralógica de los productos del beneficio
permitió concluir que estos procesos pueden afectar la
cristalinidad de los minerales. En el caso específico de la mina 4. CONCLUSIONES
Las arcillas explotadas son de origen sedimentario, de tipo
coluvial y lacustre; contienen materiales deslizados de las
Formaciones circundantes, y suprayacen a estas. Se clasifi-
caron las arcillas como illítico-caoliníticas, donde minerales
como illita, caolinita y cuarzo son los más abundantes,
presentando una mala cristalización.
La caracterización química y mineralógica permitió establecer
el tipo de proceso de beneficio, frente a problemas específicos
como, por ejemplo, la presencia de óxidos de hierro que
impregnan y colorean las arcillas.
La caracterización mineralógica de los productos del beneficio
permitió concluir que estos procesos pueden afectar la
cristalinidad de los minerales. En el caso específico de la mina 4. CONCLUSIONES
Las arcillas explotadas son de origen sedimentario, de tipo
coluvial y lacustre; contienen materiales deslizados de las
Formaciones circundantes, y suprayacen a estas. Se clasifi-
caron las arcillas como illítico-caoliníticas, donde minerales
como illita, caolinita y cuarzo son los más abundantes,
presentando una mala cristalización.
La caracterización química y mineralógica permitió establecer
el tipo de proceso de beneficio, frente a problemas específicos
como, por ejemplo, la presencia de óxidos de hierro que
impregnan y colorean las arcillas.
La caracterización mineralógica de los productos del beneficio
permitió concluir que estos procesos pueden afectar la
cristalinidad de los minerales. En el caso específico de la mina 4. CONCLUSIONES
Las arcillas explotadas son de origen sedimentario, de tipo
coluvial y lacustre; contienen materiales deslizados de las
Formaciones circundantes, y suprayacen a estas. Se clasifi-
caron las arcillas como illítico-caoliníticas, donde minerales
como illita, caolinita y cuarzo son los más abundantes,
presentando una mala cristalización.
La caracterización química y mineralógica permitió establecer
el tipo de proceso de beneficio, frente a problemas específicos
como, por ejemplo, la presencia de óxidos de hierro que
impregnan y colorean las arcillas.
La caracterización mineralógica de los productos del beneficio
permitió concluir que estos procesos pueden afectar la
cristalinidad de los minerales. En el caso específico de la mina 4. CONCLUSIONES
Las arcillas explotadas son de origen sedimentario, de tipo
coluvial y lacustre; contienen materiales deslizados de las
Formaciones circundantes, y suprayacen a estas. Se clasifi-
caron las arcillas como illítico-caoliníticas, donde minerales
como illita, caolinita y cuarzo son los más abundantes,
presentando una mala cristalización.
La caracterización química y mineralógica permitió establecer
el tipo de proceso de beneficio, frente a problemas específicos
como, por ejemplo, la presencia de óxidos de hierro que
impregnan y colorean las arcillas.
La caracterización mineralógica de los productos del beneficio
permitió concluir que estos procesos pueden afectar la
cristalinidad de los minerales. En el caso específico de la mina 4. CONCLUSIONES
Las arcillas explotadas son de origen sedimentario, de tipo
coluvial y lacustre; contienen materiales deslizados de las
Formaciones circundantes, y suprayacen a estas. Se clasifi-
caron las arcillas como illítico-caoliníticas, donde minerales
como illita, caolinita y cuarzo son los más abundantes,
presentando una mala cristalización.
La caracterización química y mineralógica permitió establecer
el tipo de proceso de beneficio, frente a problemas específicos
como, por ejemplo, la presencia de óxidos de hierro que
impregnan y colorean las arcillas.
La caracterización mineralógica de los productos del beneficio
permitió concluir que estos procesos pueden afectar la
cristalinidad de los minerales. En el caso específico de la mina 4. CONCLUSIONES
Las arcillas explotadas son de origen sedimentario, de tipo
coluvial y lacustre; contienen materiales deslizados de las
Formaciones circundantes, y suprayacen a estas. Se clasifi-
caron las arcillas como illítico-caoliníticas, donde minerales
como illita, caolinita y cuarzo son los más abundantes,
presentando una mala cristalización.
La caracterización química y mineralógica permitió establecer
el tipo de proceso de beneficio, frente a problemas específicos
como, por ejemplo, la presencia de óxidos de hierro que
impregnan y colorean las arcillas.
La caracterización mineralógica de los productos del beneficio
permitió concluir que estos procesos pueden afectar la
cristalinidad de los minerales. En el caso específico de la mina 4. CONCLUSIONES
Las arcillas explotadas son de origen sedimentario, de tipo
coluvial y lacustre; contienen materiales deslizados de las
Formaciones circundantes, y suprayacen a estas. Se clasifi-
caron las arcillas como illítico-caoliníticas, donde minerales
como illita, caolinita y cuarzo son los más abundantes,
presentando una mala cristalización.
La caracterización química y mineralógica permitió establecer
el tipo de proceso de beneficio, frente a problemas específicos
como, por ejemplo, la presencia de óxidos de hierro que
impregnan y colorean las arcillas.
La caracterización mineralógica de los productos del beneficio
permitió concluir que estos procesos pueden afectar la
cristalinidad de los minerales. En el caso específico de la mina 4. CONCLUSIONES
Las arcillas explotadas son de origen sedimentario, de tipo
coluvial y lacustre; contienen materiales deslizados de las
Formaciones circundantes, y suprayacen a estas. Se clasifi-
caron las arcillas como illítico-caoliníticas, donde minerales
como illita, caolinita y cuarzo son los más abundantes,
presentando una mala cristalización.
La caracterización química y mineralógica permitió establecer
el tipo de proceso de beneficio, frente a problemas específicos
como, por ejemplo, la presencia de óxidos de hierro que
impregnan y colorean las arcillas.
La caracterización mineralógica de los productos del beneficio
permitió concluir que estos procesos pueden afectar la
cristalinidad de los minerales. En el caso específico de la mina "La Esperanza" mejoró la cristalinidad de las illitas (pérdida de las interláminas cloríticas y vermiculíticas) y aumentó el grado de desorden cristalino de las caolinitas; el primero benéfico y el segundo no recomendable para su uso en la industria del papel.

Se determinó que las arcillas de color blanco, sin ningún tipo de manchamiento, son las que más responden al proceso de beneficio y son aptas para la elaboración de cerámicas finas. Aquellas de coloraciones rosas, cremas y pardas, con el adecuado proceso de beneficio, son igualmente útiles para la cerámica y rellenos, por ejemplo, en la fabricación del caucho.

\section{RECOMENDACIONES}

La caracterización mineralógica de depósitos arcillosos ha de hacerse siempre utilizando técnicas analíticas combinadas, ya que no existe un solo método que suministre toda la información de composición y textura en el depósito. La difractometría de rayos $\mathrm{X}$ es el análisis más completo para la identificación de minerales arcillosos, sin embargo, es necesario, frente al proceso de beneficio, conocer la relación con los otros minerales presentes en el depósito, como óxidos, silicatos, carbonatos, etc., a través de otros tipos de análisis como los ópticos.
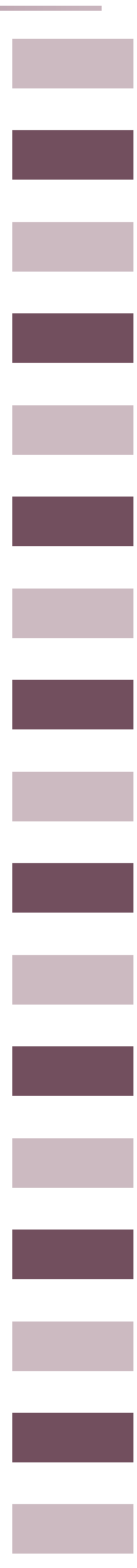


\section{REFERENCIAS}

Grim, R. (1962). Applied Clay Mineralogy. New York: Mc Graw Hill. 885 p.

Icontec (1997). Norma 2428. Especificaciones técnicas del caolín para usos industriales.

Manosalva, S. (2005). Caracterización mineralógica de arcillas, mina La Esperanza (Arcabuco, Boyacá), 136 p.
Renzoni, G. (1976). Mapa geológico plancha N. ${ }^{\circ} 171$, Escala 1:100000. Ingeominas.

Rodríguez, A. (1998). Las arcillas: aporte a su conocimiento y evaluación. Ingeominas. Informe 2304.

Thorez, J. (2003). Practical XRD Analysis of Clay Minerals. En: seminario-taller, vol. 2. Bogotá. $412 \mathrm{p}$.

Wilson, J. (1994). Industrial Clay Case Study. Indiana (USA): Exeter. $446 \mathrm{p}$. 